\title{
Electrochemical Evaluation of Geometrical Effect and Three-dimensionalized Effect of Iridium Oxide Electrodes Used for Retinal Stimulation
}

\author{
Toshihiko Noda, ${ }^{1 *}$ Yoshiko Noda, ${ }^{1}$ Po-Chun Chen, ${ }^{2}$ Makito Haruta, ${ }^{1}$ \\ Kiyotaka Sasagawa, ${ }^{1}$ Takashi Tokuda, ${ }^{1}$ Chung-Yu Wu, ${ }^{3}$ and Jun Ohta ${ }^{1}$ \\ ${ }^{1}$ Graduate School of Materials Science, Nara Institute of Science and Technology, \\ 8916-5 Takayama, Ikoma, Nara 630-0192, Japan \\ ${ }^{2}$ Department of Materials and Mineral Resources Engineering, National Taipei University of Technology, \\ 1, Sec. 3, Zhongxiao E. Rd., Taipei 10608, Taiwan, R.O.C. \\ ${ }^{3}$ Department of Electronics Engineering, National Chiao Tung University, \\ 1001 University Road, Hsinchu 30010, Taiwan, R.O.C.
}

(Received July 31, 2017; accepted October 6, 2017)

Keywords: retinal prosthesis, neural stimulation, stimulus electrode, three-dimensionalized electrode, iridium oxide

The geometry dependence and three-dimensionalized effect of the electrochemical properties of iridium oxide electrodes used in retinal prosthesis are discussed herein. Planar electrodes with various geometries were fabricated. Three-dimensionalized electrodes with microfabricated resin cores were also fabricated. The charge storage capacity (CSC) and charge injection capacity (CIC), both of which are important properties for neural stimulation, were quantitatively evaluated. This evaluation showed the important electrode property that the surface area dominantly affects the CSC. On the other hand, the CIC was not proportionally related to the electrode area, confirming that the edge region of the electrodes is significant in the mechanism of action. Moreover, performance enhancement with a three-dimensionalized electrode was clearly confirmed. The fundamental properties of highly effective stimulus electrodes were successfully evaluated and a detailed discussion is presented.

\section{Introduction}

Neural stimulation has become increasingly important in the field of brain-machine interfaces, especially for sensory prosthesis. Cochlear implants are among the most widely recognized sensory prosthesis systems. In recent years, the practical application of retinal prosthesis, a more complicated prosthesis, has been implemented. ${ }^{(1-3)}$ However, the visual quality provided to patients is insufficient because a neural interface capable of carrying huge amounts of visual information is extremely difficult to obtain. The size and number of stimulus electrodes required for the neural interface at the retina limit the practical implementation of this system. Although an electrode array comprising a huge number of microelectrodes with

*Corresponding author: e-mail: t-noda@ms.naist.jp http://dx.doi.org/10.18494/SAM.2018.1717 
dimensions similar to those of retinal cells can be fabricated by applying CMOS technology, the electrochemical performance of these electrodes does not satisfy the requirements for retinal stimulation. A critical margin of electrochemical performance must be maintained for safe stimulation, which is indispensable for implantable medical devices.

Many approaches have been investigated with the aim of achieving sufficient performance, even with miniaturized electrodes. One promising approach is to utilize capacitive electrodes ${ }^{(4-6)}$ that improve electrode performance by increasing the real surface area obtained by a micro/nano-scale surface morphology. The introduction of reactive electrodes such as oxides ${ }^{(7-9)}$ and organic polymers ${ }^{(10)}$ has also been investigated. Our focus has been on achieving high-performance iridium oxide ( $\mathrm{IrOx}$ ) materials by optimizing the fabrication process and fundamental electrochemical properties. ${ }^{(11,12)}$ The in vivo validation of neural stimulation by IrOx electrodes was also successfully achieved. ${ }^{(13)}$ Moreover, we attempted another approach for enhancing the electrochemical performance by introducing a threedimensionalized electrode. ${ }^{(14)}$ Although the potential of IrOx and the effectiveness of the three-dimensionalized electrode were demonstrated, quantitative evaluation of the electrode performance of the optimized electrode design has not yet been undertaken. Although the geometrical effect has been investigated ${ }^{(15)}$ in the case of rectangular electrodes, quantitative evaluation of the shape dependence of the electrode performance is required for optimized design of the miniaturized electrode. In addition, although Au stud bumps were used to form the three-dimensional structure, the miniaturization of that structure is unrealistic. In this study, the effects of geometrical properties, i.e., the size and shape of the electrodes, and the effect of three-dimensionalization on the electrochemical performance, were evaluated through electrochemical analysis of the electrodes. We report the fabrication of electrodes of various shapes and sizes and discuss the mechanism underlying their electrochemical properties. We also discuss the technique of fabricating a three-dimensional structure that is suitable for miniaturization. Three-dimensionalized electrodes were fabricated and electrochemical analysis was performed.

\section{Evaluation of Geometrical Effect of Planar Electrode}

Various types of planar electrodes based on IrOx were fabricated for the evaluation of the geometry dependence of the electrochemical performance. The fabricated electrodes were quantitatively characterized to discuss their electrochemical performance with the aim of optimizing the electrode design.

\subsection{Fabrication of electrodes}

Planar electrodes of various sizes and shapes were designed and fabricated. Table 1 shows a list of the fabricated electrodes. The electrodes were fabricated with circular, equilateral hexagonal, square, and rectangular geometries with aspect ratios of 4-25, as well as with an equilateral triangular shape. The surface areas of the electrodes ranged from 0.01 to $6 \mathrm{~mm}^{2}$ for each shape. Figure 1 shows the structure of a microchip with a circular electrode as one 
Table 1

List of fabricated electrodes.

\begin{tabular}{|c|c|c|c|}
\hline Shape & Length of each side/Diameter (mm) & Area $\left(\mathrm{mm}^{2}\right)$ & Perimeter length $(\mathrm{mm})$ \\
\hline \multirow{6}{*}{ Circle } & 2.38 & 4.45 & 7.48 \\
\hline & 2.13 & 3.56 & 6.69 \\
\hline & 1.13 & 1.00 & 3.54 \\
\hline & 0.56 & 0.25 & 1.77 \\
\hline & 0.34 & 0.09 & 1.06 \\
\hline & 0.11 & 0.01 & 0.35 \\
\hline \multirow{6}{*}{ Equilateral hexagon } & 1.51 & 5.92 & 9.06 \\
\hline & 1.24 & 3.99 & 7.44 \\
\hline & 0.62 & 1.00 & 3.72 \\
\hline & 0.31 & 0.25 & 1.85 \\
\hline & 0.19 & 0.09 & 1.12 \\
\hline & 0.06 & 0.01 & 0.37 \\
\hline \multirow{6}{*}{ Square } & 2.45 & 6.00 & 9.80 \\
\hline & 2.00 & 4.00 & 8.00 \\
\hline & 1.00 & 1.00 & 4.00 \\
\hline & 0.50 & 0.25 & 2.00 \\
\hline & 0.30 & 0.09 & 1.20 \\
\hline & 0.10 & 0.01 & 0.40 \\
\hline \multirow{6}{*}{ Rectangle } & $1.0 \times 6.0$ (Aspect ratio: 6$)$ & 6.00 & 14.00 \\
\hline & $1.0 \times 4.0$ (Aspect ratio: 4$)$ & 4.00 & 10.00 \\
\hline & $0.5 \times 2.0($ Aspect ratio: 4$)$ & 1.00 & 5.00 \\
\hline & $0.1 \times 2.5($ Aspect ratio: 25$)$ & 0.25 & 5.20 \\
\hline & $0.1 \times 1.0($ Aspect ratio: 10$)$ & 0.09 & 2.18 \\
\hline & $0.05 \times 0.2($ Aspect ratio: 4$)$ & 0.01 & 0.49 \\
\hline \multirow{6}{*}{ Equilateral triangle } & 3.72 & 5.99 & 11.16 \\
\hline & 3.09 & 4.13 & 9.27 \\
\hline & 1.51 & 0.99 & 4.53 \\
\hline & 0.76 & 0.25 & 2.27 \\
\hline & 0.45 & 0.09 & 1.36 \\
\hline & 0.15 & 0.01 & 0.45 \\
\hline
\end{tabular}

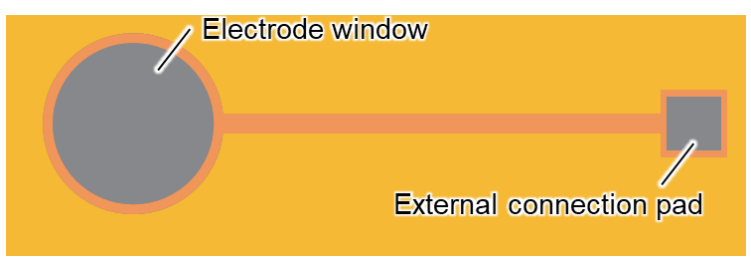

(a)

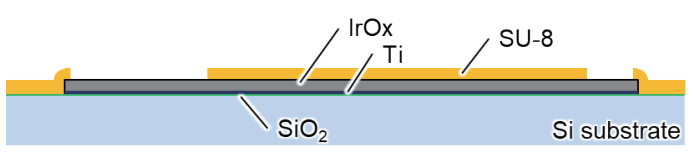

(b)

Fig. 1. (Color online) Structure of planar electrode in (a) top and (b) cross-sectional views.

example of the fabricated electrodes. The microchip had a planar thin film of IrOx, which acted simultaneously as an electrode, a lead wire, and an external connection pad on a Si substrate with a $1-\mu \mathrm{m}$-thick $\mathrm{SiO}_{2}$ surface insulation layer. The entire surface of the microchip, except for the electrode window and external connection pad, was covered with resin for insulation. 
Figure 2 shows the process flow for the fabrication of the planar electrodes. First, the surface of the $\mathrm{Si}$ substrate with a 1- $\mu$ m-thick thermal $\mathrm{SiO}_{2}$ layer was cleaned using oxygen plasma [Fig. 2(a)]. A 2- $\mu$ m-thick photoresist (AZ-5214E, Merck Performance Materials) was coated and patterned onto the substrate, as shown in Fig. 2(b). The substrate was then introduced into the sputtering chamber (CFS-4ES-II, Shibaura Mechatronics Corporation) and surface cleaning was performed by reverse sputtering. The parameters for reverse sputtering were pressure: $1 \mathrm{~Pa}$; Ar gas: $10 \mathrm{sccm}$; radio-frequency (RF) power: $50 \mathrm{~W}$; sputtering time: 1 min. A 50-nm-thick Ti film as an adhesion layer and a 1- $\mu$ m-thick IrOx film were formed by sputtering without chamber venting after the surface-cleaning process [Fig. 2(c)]. Ar sputtering was used for $\mathrm{Ti}$ deposition, and reactive sputtering with an Ir target and $\mathrm{O}_{2}$ gas was used for IrOx deposition. The parameters of Ti deposition were pressure: $0.6 \mathrm{~Pa}$; Ar gas: $20 \mathrm{sccm}$; DC power: $100 \mathrm{~W}$; sputtering time: $5 \mathrm{~min}$. The parameters for IrOx deposition were: pressure: $1 \mathrm{~Pa}$; $\mathrm{O}_{2}$ gas: $10 \mathrm{sccm}$; $\mathrm{RF}$ power: $200 \mathrm{~W}$; sputtering time: $60 \mathrm{~min}$. The sputtering parameters were the same as the optimized parameters used in our previous work. ${ }^{(14)}$ To achieve lift-off of the Ti/IrOx layer, the sputtering-deposited substrate was immersed in a solvent (AZ Remover 100, Merck Performance Materials) at $80{ }^{\circ} \mathrm{C}$ for $60 \mathrm{~min}$. The photoresist was dissolved and the Ti/ IrOx films were patterned as shown in Fig. 2(d). Finally, a permanent photoresist (SU-8 3010, MicroChem) was coated onto the surface of the microchip. The electrode window and external connection pad were opened by photolithography. Figure 3 shows the micrographs of typical fabricated electrodes.

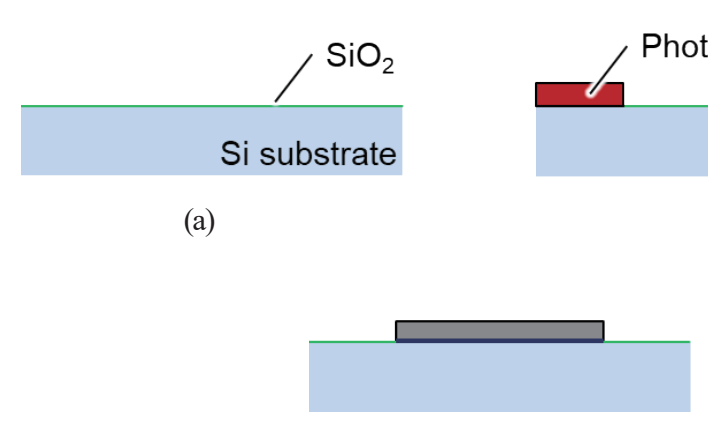

(d)

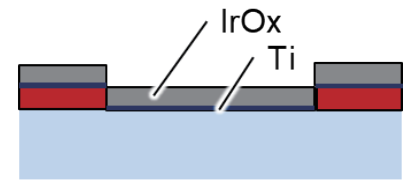

(c)

(b)

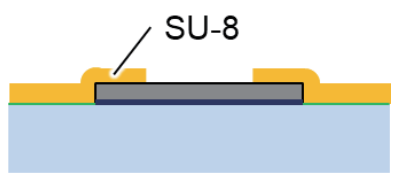

(e)

Fig. 2. (Color online) Process flow for fabrication of planar electrodes.

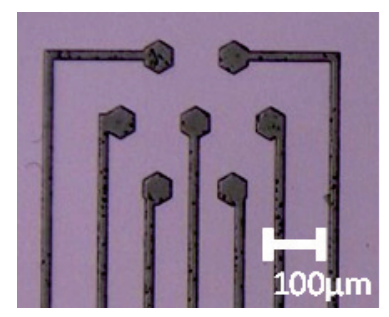

(a)

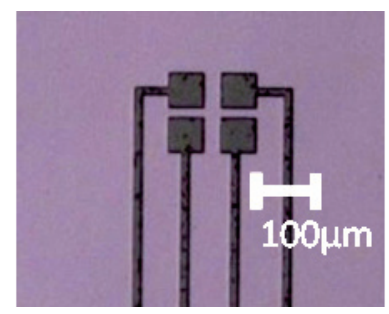

(b)

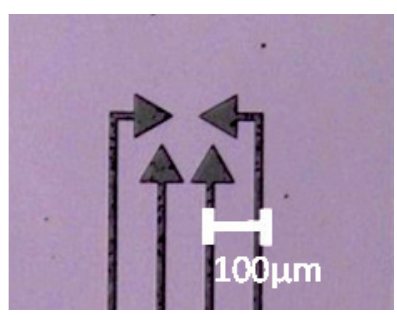

(c)

Fig. 3. (Color online) Micrographs of typical fabricated electrodes. (a) Equilateral hexagonal electrodes. (b) Square electrodes. (c) Equilateral triangular electrodes. 


\subsection{Electrochemical evaluation}

The quasi-static performance and dynamic performance, both of which are important electrochemical properties, were evaluated quantitatively. The mechanism underlying the electrochemical performance is discussed below, with the aim of optimizing the electrode design.

\subsubsection{Charge storage capacity (CSC)}

The CSC is an important parameter for evaluating the electrochemical performance of an electrode in the quasi-static state, i.e., the sufficiently slow reaction at the electrode surface. The CSC provides a good reflection of the original properties of the electrode material without complicated effects from the harmonic components of the applied voltage, charging effects, and the condition of the electrode surface. Cyclic voltammetry (CV) with a typical three-electrode configuration was performed to evaluate the CSCs of the fabricated electrodes. Phosphate buffered saline (PBS) (164-18541, $0.01 \mathrm{~mol} / \mathrm{L}$ PBS, Wako Pure Chemical Industries) was used to simulate the implanted retinal stimulator. The fabricated electrodes were used as the working electrode, a $2 \times 2 \mathrm{~cm}^{2}$ Pt plate was used as the counter electrode, and a $\mathrm{Ag} / \mathrm{AgCl}$ electrode with saturated $\mathrm{KCl}$ was used as the reference electrode; the electrodes were dipped into the PBS and connected to an electrochemical analyzer (PGSTAT204, Metrohm Autolab B.V.) that functioned as a potentiostat. The potential of the working electrode was swept from -0.65 to $0.8 \mathrm{~V}$, which corresponds to the potential window of IrOx, at a sweep speed of $50 \mathrm{mV} / \mathrm{s}$. The CSC was calculated as the integral of the cyclic voltammogram, i.e., the closed area of the CV curve. ${ }^{(16,17)}$

The surface of an Ir electrode can be activated, i.e., modified to IrOx, by the application of cyclic voltage in saline. ${ }^{(18)}$ The same behavior was observed for the sputtered IrOx film (SIROF), even though the SIROF was already oxidized. Because of the activation phenomenon, the initial condition of the as-deposited SIROF was not stable; therefore, an increase in CSC was observed. Figure 4 shows the increase in CSC during CV analysis of the circular SIROF electrode with a diameter of $1 \mathrm{~mm}$. The CSC calculation could not be performed for the first two voltage cycles

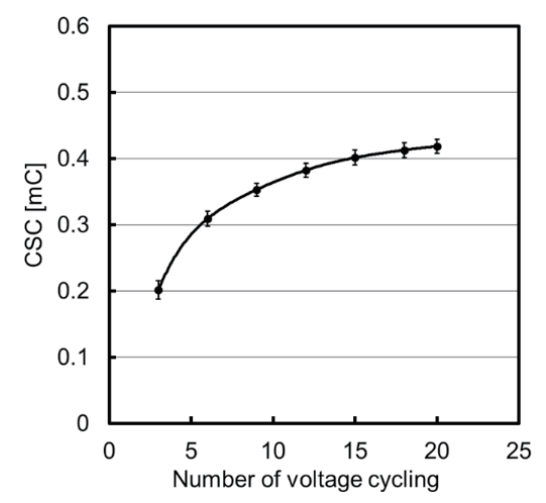

Fig. 4. Increase in CSC with voltage cycling using CV. The CSC values plotted are the average of measurements from three individual electrodes. Error bars show the standard deviations of the measured CSCs. 
because of unstable data from the CV measurement. The CSC increased in accordance with the voltage cycling after the third voltage cycle. After the 18th voltage cycle, the CSC changed by less than $1 \%$ per cycle. Therefore, in this study, the CSCs were calculated from the results of the 20th voltage cycle for stable evaluation.

CSC measurements of the fabricated electrodes were performed. At least three electrodes were analyzed for each electrode geometry. Figures 5 and 6 show CSC versus electrode area and perimeter length, respectively. All electrode shapes showed a similar trend where the CSC increased proportionally to the electrode area, i.e., the CSC was proportional to the square of the electrode perimeter length. These results show that the CSC was dominantly determined by the electrode area. Furthermore, the edge effect was not significant because the CSC was not directly proportional to the electrode perimeter length.

\subsubsection{Charge injection capacity (CIC)}

Pulsed stimulation is generally used for neural stimulation. Therefore, the electrochemical performance with the application of a pulsed voltage or current, i.e., a dynamic state, is important for practical application. In the current-controlled stimulation, the CIC was used as a performance indicator. The transient response of the electrode potential with the pulsed current that was used for retinal stimulation was measured to evaluate the CICs of the fabricated electrodes. The CIC can be determined from the maximum injectable current without exceeding the potential window of IrOx, i.e., from -0.65 to $0.8 \mathrm{~V}$. Similar to the case described in Sect. 2.2.1., a three-electrode configuration and PBS were used for the evaluation. A controlled biphasic pulse current generated by an electric stimulator with a stimulus isolator (SEN-3401 and SS-203J, Nihon Kohden Corporation) was applied between the fabricated electrode and the counter electrode. A charge-balanced anodic-first biphasic pulse with a pulse

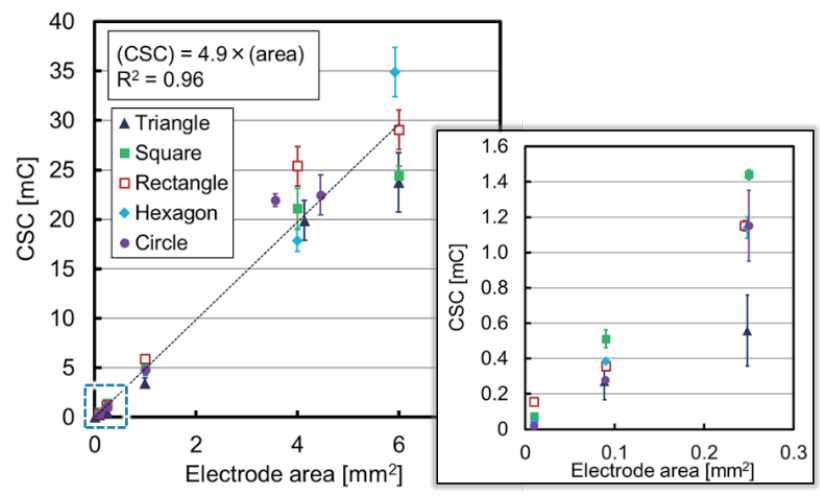

Fig. 5. (Color online) CSC vs electrode area. The plotted CSCs are average values. Error bars show the standard deviations of the CSCs. The dashed line shows the linear fit to the plot. The inset shows a magnified plot of small electrode area.

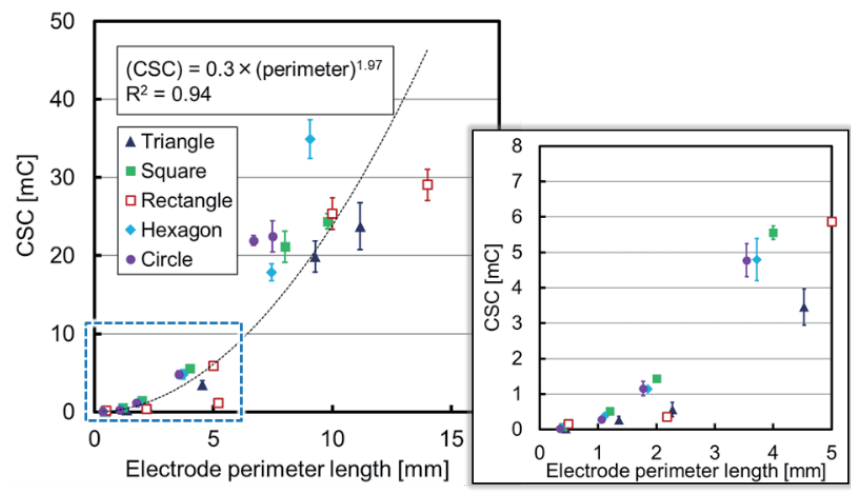

Fig. 6. (Color online) CSC vs electrode perimeter. length. The plotted CSCs are average values. Error bars show the standard deviations of the CSCs. The dashed line shows the exponential fit to the plot. The inset shows a magnified plot of small perimeter length. 
duration of $500 \mu \mathrm{s}$, an interpulse duration of $30 \mu \mathrm{s}$, and a repetition frequency of $1 \mathrm{~Hz}$ were used for the CIC evaluation. The potential of the fabricated electrode versus the reference electrode was measured using an electrochemical analyzer (PGSTAT204, Metrohm Autolab B.V.) as an electrometer.

The CIC evaluation of the fabricated electrodes was performed by averaging the values for at least three electrodes with the same geometry. The CIC is plotted versus the electrode area and electrode perimeter length in Figs. 7 and 8, respectively. By performing an approximate curve fitting, the CIC was found to be linearly related to the electrode perimeter length. However, it was found to be exponentially related to the electrode area (the exponent is approximately 3/4) rather than linearly related. The data indicate that the CIC was more sensitive to the electrode perimeter length than to the electrode area. Although further discussion is required to clarify the mechanism underlying this phenomenon, one possibility is that in the CIC measurement, which used a stimulus pulse as in practical application, high-speed charge injection was required at the interface between the electrode and the electrolyte because the stimulus pulse had high-frequency components. In some cases, an electrochemical reaction is rate-limited by the diffusional supply of ions in the electrolyte. Thus, charge injection becomes dominant because ions are supplied sufficiently at the edge of the electrode by diffusion compared with the center region of the electrode.

Here, we focused on the unit-area performance of the electrode. In the case of the IrOx electrode, which had an equivalent or smaller geometry than the diffusion layer of the electrolyte, the CIC per unit area increased owing to sufficient transport of ions. ${ }^{(17)}$ Figure 9 shows the CIC per unit area versus the electrode area. Most of the fabricated electrodes, except for the electrodes with electrode area around $0.25 \mathrm{~mm}^{2}$, were in the performance range of $100-200 \mathrm{nC} / \mathrm{mm}^{2}$. Although some of the small electrodes showed high CIC values per unit area, no clear trend of the improvement of CIC per unit area in accordance with electrode miniaturization was observed. The area of the electrodes used in this study was over 10 times

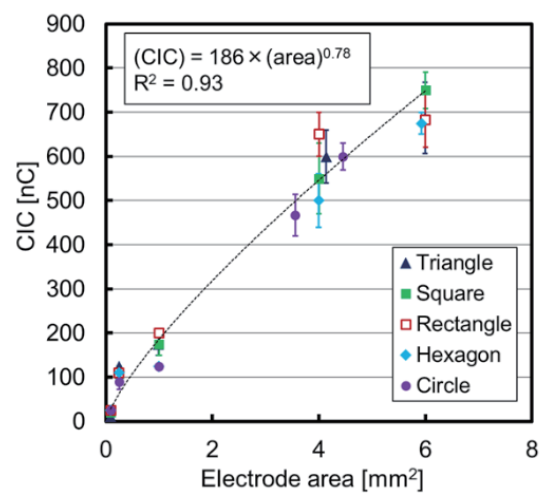

Fig. 7. (Color online) CIC vs electrode area. The CIC values are average values. Error bars show the standard deviations of the CICs. The dashed line shows the exponential fit to the plot.

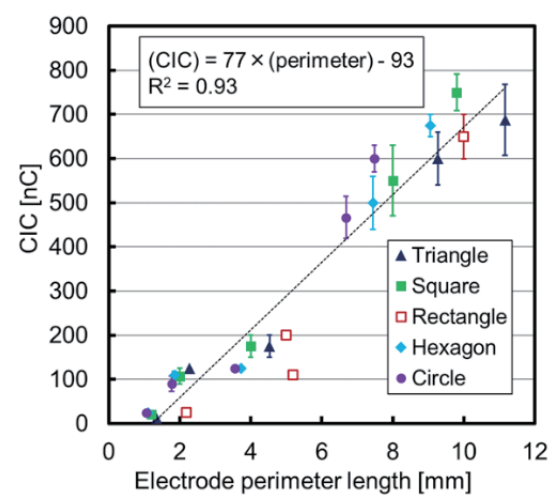

Fig. 8. (Color online) CIC vs electrode perimeter length. The CIC values are average values. Error bars show the standard deviations of the CICs. The dashed line shows the linear fit to the plot. 


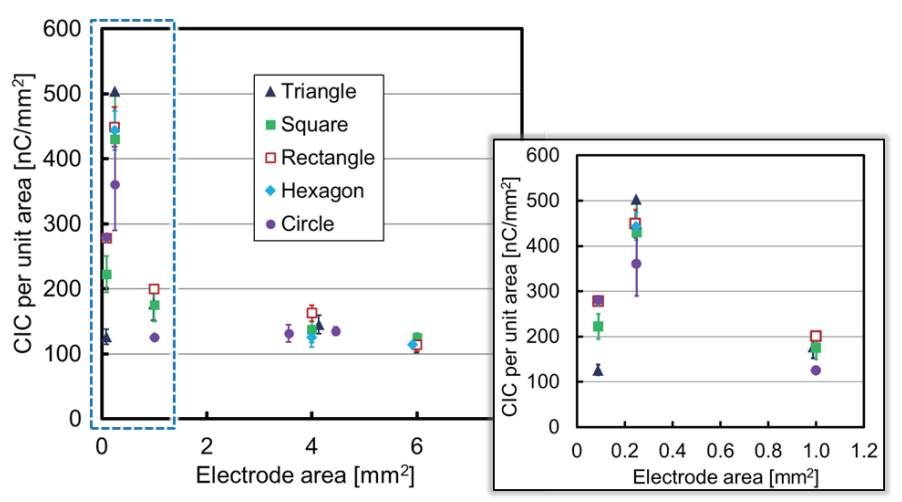

Fig. 9. (Color online) CIC per unit area vs electrode area. The CIC values are average values. Error bars show standard deviations of the CICs. Inset shows magnified plot of small electrode area.

larger than the electrode area used by Slavcheva et al. ${ }^{(16)}$ Shape dependence may become clear with electrode areas less than $0.1 \mathrm{~mm}^{2}$, which is a topic of future study.

\section{Evaluation of Three-dimensionalized Effect}

In our previous work, we fabricated three-dimensionalized electrodes using Au stud bumps as the core structure of the electrodes. ${ }^{(14)}$ The electrodes were successfully developed and showed enhanced electrochemical performance. For retinal prostheses, miniaturization of the stimulus electrodes is required to achieve high resolution, but the miniaturization of stud bumps is unrealistic. Therefore, we proposed another approach that is suitable for miniaturization by photolithography in order to form a three-dimensional core structure. In this section, two types of three-dimensionalized electrodes fabricated by the proposed approach are discussed on the basis of the electrochemical evaluation.

\subsection{Fabrication of electrodes}

Photolithography is suitable for microfabrication in batch processes with high relative accuracy. To achieve the proposed fabrication of a three-dimensional structure by photolithography, two types of three-dimensionalized electrodes with epoxy resin (permanent photoresist) cores were fabricated to evaluate the three-dimensionalized effect. The first type was a monoblock electrode with a cuboidal morphology $\left(400 \times 400 \times 70 \mu \mathrm{m}^{3}\right)$ in a 500 $\times 500 \mu \mathrm{m}^{2}$ electrode window. The second type was a multibump electrode with a $5 \times 5$ array of bumps $\left(50 \times 50 \times 10 \mu \mathrm{m}^{3}\right)$ in the electrode window. A planar electrode with the same dimensions in the electrode window was also fabricated. The surfaces of these electrodes comprised IrOx. A planar Pt electrode was also fabricated as a reference.

Three-dimensionalized electrodes were fabricated by the process shown in Fig. 10. A Si substrate with a thermal $\mathrm{SiO}_{2}$ layer was used, as described in Sect. 2. First, three-dimensional cores of epoxy resin were formed on the substrate by photolithography, as shown in Fig. 10(b). SU-8 3050 (MicroChem) was used for the monoblock electrodes and SU-8 3010 (MicroChem) 


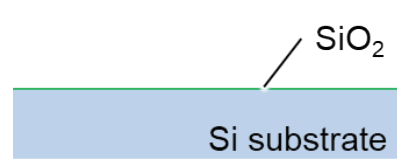

(a)

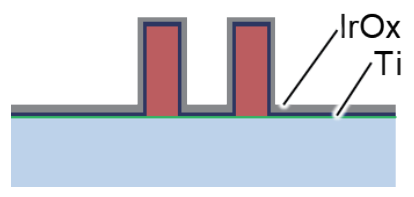

(c)

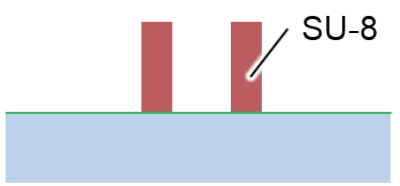

(b)

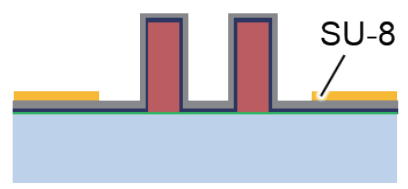

(d)

Fig. 10. (Color online) Process of fabricating three-dimensionalized electrode.

was used for the multibump electrodes. Ti/IrOx was then deposited on the substrate with a three-dimensional structure. The deposition and sputtering parameters were the same as those used in the fabrication process described in Sect. 2.1. Finally, the electrode window was formed on the substrate by coating with SU-8 3010. Figure 11(a) shows a scanning electron microscopy (SEM) image of the monoblock electrode and Fig. 11(b) shows SEM images of the multibump electrode.

\subsection{Electrochemical evaluation}

The fabricated electrodes were evaluated in a manner similar to that used for the planar electrodes described in Sect. 2.2. The CSC and CIC values were measured and the mechanism underlying the three-dimensionalized effect is discussed below.

\subsubsection{CSC}

The CSCs of the fabricated electrodes were evaluated using the same configuration and parameters as listed in Sect. 2.2.1. The geometric surface areas of the three-dimensionalized electrodes were calculated as per the designed dimensions while neglecting any error from the fabrication process. As shown in Fig. 12, the relationship between the geometric surface area and the CSC of the IrOx electrodes was proportional, irrespective of the electrode morphology, i.e., planar or three-dimensional. This property is similar to the shape dependence described in Sect. 2.2.1. Although ion diffusion and the electric field can be considered nonuniform at the edges compared with at the center of the three-dimensional structure, the edge effect did not significantly affect the CSC.

\subsubsection{CIC}

The CICs of the fabricated electrodes measured with an anodic-first (AF) biphasic pulse and a cathodic-first (CF) biphasic pulse were evaluated as described in Sect. 2.2.2. The results are shown in Fig. 13(a) for the AF pulse and in Fig. 13(b) for the CF pulse. The dashed lines in Fig. 


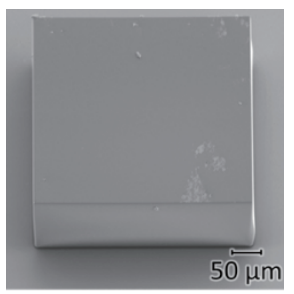

(a)

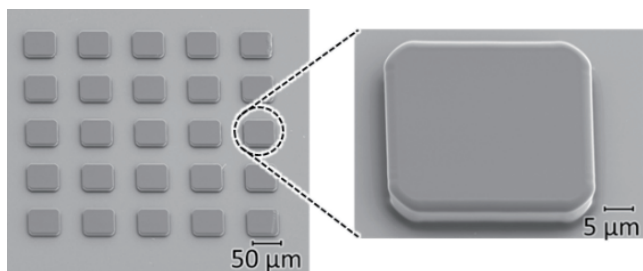

(b)

Fig. 11. SEM images of three-dimensionalized electrodes. (a) Monoblock and (b) $5 \times 5$ multibump electrodes. The inset shows the magnified SEM image of a single bump.

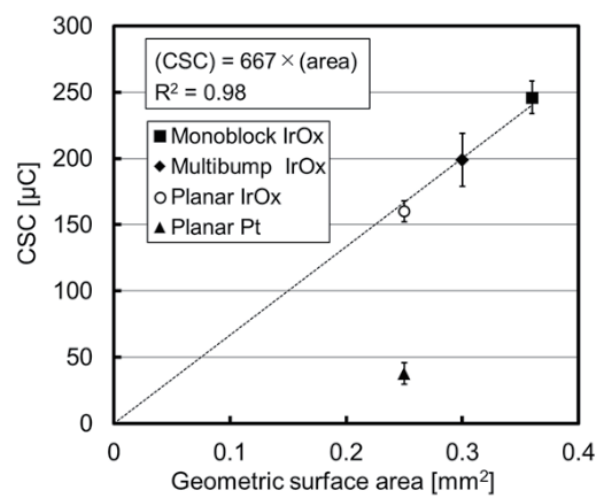

Fig. 12. CSC of fabricated electrodes. Error bars show the standard deviations. The dashed line shows proportional fitting.

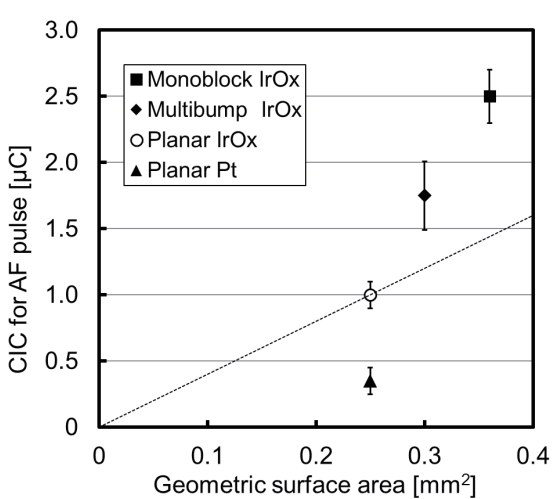

(a)

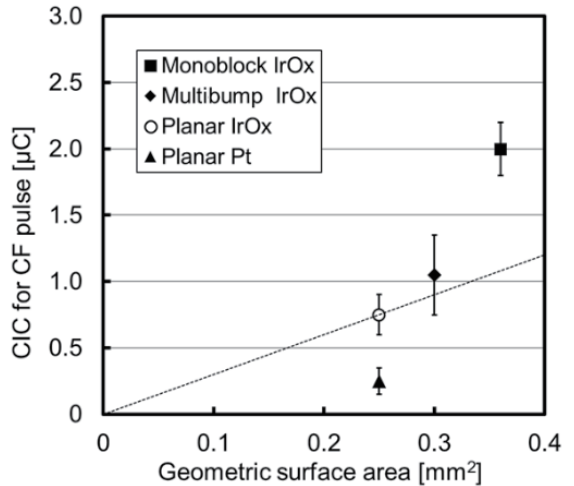

(b)

Fig. 13. CIC of fabricated electrodes. CIC for AF pulses is shown in (a) and CIC for CF pulses is shown in (b). Error bars show the standard deviations. The dashed lines show the proportionality based on the planar IrOx electrode.

13 are proportionality lines based on the planar IrOx electrode. When the CICs of the threedimensionalized electrodes were proportional to the geometric surface area, the measured CICs could be plotted on the dashed line. However, the CICs of the three-dimensionalized electrodes were much larger than those expected from a proportional relation to the geometric surface 
area. These results mean that the three-dimensionalized electrodes had a CIC enhancement effect derived from both the expansion of the geometric surface area and another mechanism. Other types of three-dimensionalized electrodes should be fabricated and evaluated to enable further detailed discussion. One possible reason for CIC enhancement effect of the threedimensionalized electrode might be that the CIC behavior was the same as that of the planar electrodes discussed in Sect. 2.2.2. The ion supply by diffusion in the electrolyte became highly efficient on the convex morphology of the three-dimensional structure.

\section{Conclusions}

The mechanism underlying the electrochemical performance of an IrOx electrode intended for retinal prosthesis was discussed. Planar IrOx electrodes with various geometries were fabricated. The CSC was found to be dominantly determined by the electrode area. However, the perimeter length strongly affected the CIC. No significant dependence on the electrode geometry was confirmed. Three-dimensionalized electrodes with a core structure fabricated by photolithography were proposed. The fabricated electrodes showed CIC enhancement that exceeded the surface area expansion because of three-dimensionalization.

The fundamental mechanism underlying the electrochemical performance was elucidated in this study. The obtained knowledge could be utilized to optimize electrode design to achieve maximum performance, even in miniaturized electrodes. Although electrodes on the micrometer, sub-micrometer, and nanometer scale were not evaluated in this study, as they are a topic for further study, their performance might be of interest to investigate because the edge effect and nonuniform diffusion of ions was prominent for electrodes with geometries on those scales.

\section{Acknowledgments}

This work was partially supported by the Japanese-Taiwanese Cooperative Program on "Bioelectronics" and "Biophotonics" from the Japan Science and Technology Agency (JST), and by a research grant from the Murata Science Foundation, Japan.

\section{References}

1 T. Fujikado, M. Kamei, H. Sakaguchi, H. Kanda, T. Morimoto, Y. Ikuno, K. Nishida, H. Kishima, T. Maruo, K. Konoma, M. Ozawa, and K. Nishida: Invest. Ophthalmol. Visual Sci. 52 (2011) 4726.

2 Y. H.-L. Luo and L. da Cruz: Prog. Retin. Eye Res. 50 (2016) 89.

3 K. Stingl, K. U. Bartz-Schmidt, D. Besch, C. K. Chee, C. L. Cottriall, F. Gekeler, M. Groppe, T. L. Jackson, R. E. MacLaren, A. Koitschev, A. Kusnyerik, J. Neffendorf, J. Nemeth, M. A. N. Naeem, T. Peters, J. D. Ramsden, H. Sachs, A. Simpson, M. S. Singh, B. Wilhelm, D. Wong, and E. Zrenner: Vision Res. 111 (2015) 149.

4 B. Ilic, D. Czaplewski, P. Neuzil, T. Stanczyk, J. Blough, and G. J. Maclay: J. Mater. Sci. 35 (2000) 3447.

5 Y. Terasawa, H. Tashiro, Y. Nakano, K. Osawa, and M. Ozawa: Invest. Ophthalmol. Visual Sci. 54 (2013) 1054.

6 M. Janders, U. Egert, M. Stelzle, and W. Nisch: Proc. 18th Annu. Int. Conf. IEEE Engineering in Medicine and Biology Society (IEEE, 1996) 245.

7 L. S. Robblee, J. L. Lefko, and S. B. Brummer: J. Electrochem. Soc. 130 (1983) 731.

8 S. F. Cogan, T. D. Plante, and J. Ehrlich: Conf. Proc. IEEE Engineering in Medicine and Biology Society 6 (2004) 4153. 
9 S. F. Cogan, P. R. Troyk, J. Ehrlich, and T. D. Plante: IEEE Trans. Biomed. Eng. 52 (2005) 1612.

10 S. Venkatraman, J. Hendricks, Z. A. King, A. J. Sereno, S. Richardson-Burns, D. Martin, and J. M. Carmena: IEEE Trans. Neural Syst. Rehabil. Eng. 19 (2011) 307.

11 Y. L. Pan, T. Noda, K. Sasagawa, T. Tokuda, H. Kanda, T. Fujikado, and J. Ohta: J. Phys. Conf. Ser. 352 (2012) 12005.

12 Y. L. Pan, T. Noda, K. Sasagawa, T. Tokuda, and J. Ohta: IEEJ Trans. Electr. Electron. Eng. 8 (2013) 310.

13 T. Noda, K. Sasagawa, T. Tokuda, Y. Terasawa, H. Tashiro, H. Kanda, T. Fujikado, and J. Ohta: Sens. Actuator, A 211 (2014) 27.

14 T. Fujisawa, T. Noda, M. Hayashi, R. Kobe, H. Tashiro, H. Takehara, K. Sasagawa, T. Tokuda, C. Y. Wu, and J. Ohta: Sens. Mater. 28 (2016) 1303.

15 S. F. Cogan, J. Ehrlich, and T. D. Plante: Proc. 36th Annu. Int. Conf. IEEE Engineering in Medicine and Biology Society (IEEE, 2014) 6850.

16 E. Slavcheva, R. Vitushinsky, W. Mokwa, and U. Schnakenberg: J. Electrochem. Soc. 151 (2004) E226.

17 S. F. Cogan: Annu. Rev. Biomed. Eng. 10 (2008) 275.

18 A. Hung, I. B. Goldberg, and J. W. Judy: Implantable Neural Prostheses 2, Techniques and Engineering Approaches (Springer, New York, 2009) 191. 